Review

\title{
Why the Quantum Brain?
}

\author{
Sergey B. Yurchenko *
}

Independent Research Center, P.O. 710132, Andijan, Uzbekistan; E-Mail: s.yucko@gmail.com

* Correspondence: Sergey B. Yurchenko; E-Mail: s.yucko@gmail.com

Academic Editor: Raul Valverde

Special Issue: Quantum Brain Dynamics

OBM Neurobiology

2021, volume 5, issue 3

doi:10.21926/obm.neurobiol.2103103
Received: May 25, 2021

Accepted: June 27, 2021

Published: July 14, 2021

\begin{abstract}
This article reviews the modern approaches to the quantum brain hypothesis. The aim is to consider the hypothesis and its classical brain-machine alternative from a broad perspective, including physics, biology, computer science, cosmology, and metaphysics. My starting point is that asking whether consciousness can or cannot have free will is fundamentally incorrect. This aspect is challenged by both physics and neuroscience. The paper argues that the search for conscious free will, as it is typically tested in Libet-type experiments, implies putting the cart before the horse. From the evolutionary perspective, a more correct question is this. Might primitive neural networks of simple organisms have possessed free volitional mechanisms (quantum in origin) as an extremely valuable acquisition for the flourishing of life? Might then the mechanisms have evolved from primary (rapid and random) reflexes in the oldest brain regions such as the brainstem to give rise to conscious cortex-centered properties in later stages of the brain evolution?
\end{abstract}

\section{Keywords}

Quantum brain; free will; consciousness; superdeterminism; criticality; exocytosis

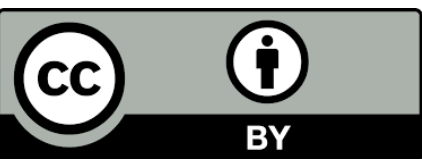

(C) 2021 by the author. This is an open access article distributed under the conditions of the Creative Commons by Attribution License, which permits unrestricted use, distribution, and reproduction in any medium or format, provided the original work is correctly cited. 


\section{Introduction}

Today quantum effects have been increasingly detected in biology [1-4] as opposed to the expectations that coherence would be rapidly destroyed in too hot biological processes, and quantum computations should be thermalized as noise. Experiments have demonstrated that a warm wet environment of cellular structures can be conducive to quantum coherence and processing key quantized events to larger spatiotemporal scales. Several sensory systems in both vertebrates and invertebrates have evolved signal detection capabilities that can amplify the energy of a single photon $[5,6]$. Yet, there is evidence that in vivo nanostimulations of a single neuron can cause neuronal avalanches over brain networks [7-9], thereby affecting cognitive and behavioral domains $[10,11]$. Nonetheless, there is no direct evidence that quantum effects can affect brain dynamics in operational ways to generate hybrid quantum/classical computations (for review, see [12]). Whether those effects are involved with consciousness remains difficult to test experimentally. Hence the question: Why the quantum brain?

A common feature of quantum-inspired approaches to consciousness is the involvement of entanglement and nonlocality. For example, Rosu [13] argues "It might well be that a biological computer makes use of EPR-type correlations; thus, promoting itself to a superior level of existence." Another known advocate of the quantum brain/mind program, Georgiev [14], suggests seven long-standing problems of consciousness that seriously clash with the principles of classical physics: the physical boundary problem, the binding problem, the causal potency problem, the free will problem, the inner privacy problem, and the hard problem.

Typical explanations aim to account for the synchronization of neural processes across different brain regions that might make the brain intelligent. This is called a binding problem in neuroscience. It is striking that quantum coherency and entanglement should be recruited to provide nonlocal binding of sensory and cognitive brain networks in processing unitary conscious self [15], as if no classical neural processes, for example, those of Coordination dynamics of coupled phase oscillators [16] in the Langevin formalism, might do it as well.

Thus, one may ask: Why a conscious quantum computer would be more evolutionarily preferable than a conscious classical computer [17]? The only reason to assume quantum brain hypotheses, such as the Hameroff-Penrose microtubule computations in the dendrites [18] or the Beck-Eccles quantum trigger in a synaptic cleft [19] or entanglement processed with nuclear spin in Posner molecules [20], is free volition that should be protected against determinism and/or a classical stochastic account of brain dynamics. Here "free volition" means the ability of the brain (not necessarily consciousness) to make a choice that is not completely predetermined from the past. My goal is to emphasize what can be said unambiguously in favor of the quantum brain program, regardless of any experimental evidence that may or may not be obtained in the future.

I am not going to speculate on the quantum brain hypotheses involving superposition, entanglement, and nonlocality in making consciousness and cognition. Instead, I argue that quantum indeterminism is the only necessary phenomenon that must be required by any adequate theory of brain dynamics to account for various volitional mechanisms such as active inference in Bayesian brain dynamics, self-initiated action in Libet-type experiments, or cognitive control in psychological studies, all underlying conscious states as these unfold over time in neural information-processing of the brain. Thus, I will focus on a causal account of brain dynamics and, more generally, the nature of consciousness in the universe. 
This paper argues that non-trivial quantum contributions to brain dynamics are necessary to account for the valuable "commodity" [21] in human practice, commonly labeled as "free will." Importantly, it seems futile to defend conscious "compatibilist" will as a self-initiated action that could have mental power over underlying neural activities. Such mental power could be justified only by appealing to Cartesian dualism. This kind of volition is abundantly disproved by many Libettype experiments e.g., [22-24]. My aim is to defend the free cognitive evolution of the brain over the lifespan. The brain evolves neurophysiologically by accumulating the information encoded uniquely in its memory networks from birth. In this way, the brain also evolves anatomically due to neuroplasticity, creating new and more effective connectivity patterns and functional modules across brain regions.

To provide the free cognitive evolution, the brain should be able to make a free choice employed by different volitional-cognitive processes, such as active inference, action selection, attentional effort, embodied choice, and cognitive control. These are crucial in Bayesian brain models; however, no underlying free volitional mechanism is suggested there. Should we then seriously assume that computational Al systems if exploiting Bayesian learning could spontaneously acquire free will and even consciousness? Many neuroscientists and psychologists maintain this idea of mechanistic consciousness causally processed in the brain, and discuss how much of those causal processes are captured by Bayesian statistical models. The discussion is based on the fact that at a level most relevant to neuroscience, all neural processes are classical and, thus, completely predetermined from the past. A complete causal model of the brain as a physical body governed by deterministic laws is viewed by them as a final theory of consciousness. Many proponents of such a theory have underestimated its logical consequences. Not only conscious will but any free volitional mechanism should be impossible there. On this condition, the cognitive brain evolution should be completely predetermined from the past.

Predetermination from the past will result in the following two fundamental consequences. The first, called here "weak consequence", underlies the brain-machine metaphor and forms the basis for artificial consciousness, welcomed by many scientists. Moreover, not only artificial consciousness but also any particular consciousness could be copyable to run automatically on many digital clones simultaneously. Yet, there is a "strong consequence" known as superdeterminism in physics. Superdeterminism is a hypothesis that the universe is a computation (in principle, reversible) going on its own by symmetric laws of Nature. The future emerges unambiguously from the past, and no one could do something freely, randomly, or by chance because everything in the universe is causally predetermined from a distant past, ultimately in the initial conditions of the Big Bang.

However, on the assumption of Big Bang cosmology combined with the second law of thermodynamics and the so-called anthropic arguments, superdeterminism leads us inevitably to the scenario of a designed clockwork universe [25]. Thus, the strong consequence of predetermination is no more than a scientifically rigorous version of old-fashioned fatalism and Newtonian deism. Surprisingly, among the proponents of this scenario happen (consciously or not) Bohm [26] with his Wholeness and Implicate Order, 't Hooft [27] with his Cellular Automata, and Everett [28] with Many Worlds. 


\section{Quantum Mechanical Interpretations}

All quantum brain dynamics models are naturally inspired by various interpretations of quantum mechanics (QM), each with its own view on the nature and role of consciousness as related to observers in terms of the infamous "measurement problem" causing a collapse of the wave function $\psi(t)$ at a moment of measurement. The collapse is observed as a discontinuous violation of the unitary evolution of a quantum system governed by the Schrödinger equation and resulting in a random outcome. The Copenhagen "naïve" interpretation suggested that the wavefunction is a probability function so that the collapse can be explained as an effect of acquiring new information in terms of Bayesian updating. This has nothing to tell us about a more fundamental reality.

First of all, I would like to divide QM interpretations into epistemic and ontic ones presented in terms of observer-independent and observer-dependent views. The epistemic position holds that quantum formalism is merely a tool for deriving statistical predictions over the underlying completely deterministic reality. In this sense, the Copenhagen interpretation is apparently $\psi$ epistemic as a necessary response to the probabilistic description of quantum mechanics, resulting eventually in a random outcome of the measurement. The hidden variable(s) theory had been suggested to underpin the Copenhagen view and restore determinism. The hidden variable theories are of two kinds with local or nonlocal variables. What is of great importance for us is that they all are deterministic in nature. For example, Bohmian mechanics [26] with the superluminal pilot-wave is nonlocal, whereas the Cellular Automaton Interpretation (CAI) of ' $t$ Hooft [27] postulates all physical interactions to be local in the "ontological" Planckian basis. Both these rule out any free volitional mechanism in the brain, and, therefore, have to be rejected by us with respect to the latter.

The hypothesis that the brain is totally controlled by the hidden deterministic variables was suggested by Bell [29] under the name "superdeterminism" (although he had dismissed the idea in favor of free will). One of the assumptions underlying the free will debates, namely the "responsiveness assumption" holds in accordance with people's commonsense that a subject's free decision has to be made for a reason, not randomly. Nevertheless, it happens that within the physical cause-effect paradigm, only a random element independent of the antecedent brain processes might account for genuine free will in contrast to classical processes, leaving a loophole for superdeterminism. The term "randomness" used throughout probabilistic descriptions is epistemic as it depends on an individual's state of knowledge that might in principle be refined. In contrast, quantum mechanics is irreducible to any underlying more detailed deterministic specification, just as it follows from quantum no-go theorems [29, 30]. So, in quantum information science, effects of entanglement and superposition are widely used in cryptographic applications to generate Bell-certified random numbers that could not be prepared classically [30].

I replace the Copenhagen "naïve" orthodoxy with Heisenberg's metaphysical cut between the underlying (potential) reality and the classical (actual) world of observed facts [31]. Thus, I relate this duplex world to a $\psi$-epistemic view. Unfortunately, metaphysics is always more ambiguous than a pure operational framework within which science resides. As Heisenberg had drawn considerable attention to consciousness and free will, placing them in his ontological basic world of potentialities, his view underpinned by the uncertainty principle was rather $\psi$-ontic and thus beneficial to free volition. Such a position had just been initiated by Whitehead [32] in his metaphysics of "becoming," which was later maintained by Penrose [18] and Stapp [33] in their 
quantum brain models that are based on a $\psi$-ontic interpretation, emphasizing a fundamentally random aspect of the physical world.

Similarly, Wheeler follows von Neumann [34] and maintains the $\psi$-ontic position [35]. The mindbrain dualism of Neumann-Wheeler is based on psychophysical parallelism as a scientific principle, dividing the world into an observed system and the observer, i.e., consciousness properly. Instead, Bohm [26] speculates on the hidden deterministic variables in QM formalism. In Bohmian mechanics, a superluminal pilot-wave is introduced to describe quantum mechanical interference processes, thereby circumventing both Copenhagen probabilistic interpretation and consciousnessinduced wavefunction collapse in the Neumann-Wheeler view. In Bohm's nonlocal wholeness, consciousness emerges from the implicate order in a superdeterministic manner as a part of the universe and having no free will but acting as a neural marionette of the order.

Surprisingly, Bohm had thought to put his interpretation in favor of consciousness by replacing quantum randomness with superdeterminism [36]. The main distinction one might then find between his wholeness and Everett's multiverse is nonlocality. While Bohmian interpretation requires superluminal signaling, the "Many Worlds" interpretation is a local theory by removing the wavefunction collapse from the deterministic unitary evolution of the Schrödinger equation. Instead, consciousness (observer) is split into many copies, each placed into a separate superdeterministic world with its particular destiny.

According to the decoherence theory, the wavefunction collapse does not depend directly on an observer, the observer (consciousness) is only a part of the environment playing the role of an objective "witness" for abundant information about a quantum state [37, 38]. The process by which the fittest information is propagated through the environment, at the expense of incompatible information, is called quantum Darwinism (QD). This can account for the objective reality of the classical events that observers indeed see behind themselves in the past as emerging post-factum from the quantum world containing redundant histories (potentialities), only one of which will be recorded in the environment. Thus, QD avoids Neumann-Wheeler dualism, Everett's Many Worlds, splitting consciousness into numberless parallel realities, and Bohm wholeness guided by a superdeterministic order. The general design of QD can be well applicable to brain dynamics in the sense that consciousness emerges from multiple neural activities occurring simultaneously and bound together as unitary macrostates that are subjective perceived as a consistent stream of consciousness excluding dual personality [39].

\section{Quantum Brain Models}

A covert idea underlying quantum brain approaches is that if the world could be observerdependent, then brain dynamics should also be dependent on consciousness as soon as the brain is a part of the physical world. But if causal potency of consciousness is viable, where does the conscious observer reside in space to be separable from the brain even for a moment? To put it differently, is the brain a part of the environment that consciousness can influence in the same manner as it does to the external world? In fact, such a separation of consciousness from neural activities is an "original sin" of many if not all, quantum-inspired models that are covertly drawn from the philosophy of mind and human pervasive belief that they are conscious agents endowed with mental control over brain dynamics and ability to choose freely which actions to perform. 
The quantum brain hypothesis holds that quantum mechanical effects can play a decisive role in brain dynamics for consciousness emergence, despite the fact that the level of neuroscience all meaningful neural processes contributing to mental properties are apparently classical, like those exploited in computers. Then why would a conscious quantum computer have any advantage over a conscious classical computer? Before answering this question one might ask: Why should a classical computer be in general conscious? What principled distinctions could be found between deterministic information-processing machines and conscious brains? At least, one distinction we grant to brains is free will, the ability to do otherwise. In other words, we believe that the stream of consciousness is not causally predetermined from the past, thereby making conscious beings free of predetermination, unlike machines.

In fact, the only reason to welcome the quantum brain hypothesis is superdeterminism forbidding free will and, in general, any sort of randomness in the universe. This is not to say "quantum mechanics is mysterious, consciousness is also mysterious, and hence they both must be involved" [17]. Rather, the argument can go as follows. First, only quantum effects are genuinely random, not merely epistemic as if depending on our incomplete knowledge about underlying physical processes. Second, randomness, as being the only legitimate candidate for indeterminism, should have something to do with our understanding of free will and moral responsibility.

This argument is often referred to the fact that consciousness is unobservable, just as quantum states [14]. Indeed, the no-cloning theorem [30] prohibits learning and copying an unknown quantum state because its "inner privacy" is protected from uncovering by a random collapse. Consciousness holds the same privacy self-evidential to humans by the Cartesian cogito. So, in modern neuroscience, there exists no other strict criterion to verify conscious presence than selfevidence, resulting in the ability of a subject to report on their states. Thus, just this secured privacy of the quantum world would prevent us from uncovering the mystery of consciousness as a "thingin-and-for-itself."

Of course, this sounds too opaque to understand how consciousness should depend upon quantum mechanics. The famous observer-dependent phenomena are of little use here unless dualism applies. Nonetheless, this can be put towards an operational way. Instead, suppose there is a neurophysiological free volition mechanism placed in the brainstem, the oldest part of the brain responsible for automatic brain functions and reflexes. Let us assume that the mechanism is quantum in origin, which random outcome can be amplified by neuronal avalanches across many spatiotemporal (classical) scales and, at the same time, constrained and processed by cognitive networks of the thalamocortical system to a macrostate of the brain associated with consciousness emergence. This conscious state, as being caused by a key quantized event in the brain, should not have been completely predetermined from the past as if the brain had free volition to generate that conscious state but not another. Although it would leave no room for conscious free will, consciousness itself, emerging from brain dynamics, might not be copyable to run in parallel on many digital clones. Thus, the "mystery" of consciousness acquires an unambiguous sense of nocloning.

\subsection{Penrose-Hameroff Model}

Among quantum brain hypotheses, the orchestrated objective reduction (Orch OR) scenario of Penrose and Hameroff [18] suggests a more detailed approach to quantum brain dynamics. This 
starts with the idea that the brain is not a Turing machine according to the Gödel theorem. Penrose stresses on an incomputable aspect of human thinking and conscious experience. Indeed, humans are able to lightly understand logical paradoxes, for example, the Liar paradox that is very similar in construction to the Gödel theorem on the incompleteness of formal systems such as Peano arithmetic. Those might be consistently operated by a Turing machine, except for a self-referential sentence on which the theorem is based.

However, not only digital computers but also many animals, including dolphins, elephants, and higher apes, might not understand the Liar paradox. Does it imply that human brains responsible for generating consciousness and logical reasoning differ fundamentally from animal brains? How might it be possible on the evolutionary scale where the former should have naturally evolved from the latter by inheriting their biological properties and anatomical organization? There is no evidence in neuroscience that human brains have any substantial distinction from mammal and avian brains. Thus, if we do not mystify human consciousness as opposed to simpler species, the main premise of the Orch OR does not meet the evolutionary argument.

Of course, one can assume that animals are able to perform sophisticated mental operations, for example, in bistable perception as it is assumed in quantum-like cognition models [40, 41]. Quantum cognition theorists speculate that the brain exploits quantum (non-distributive) logic rather than classical (Boolean) logic in decision-making and learning $[42,43]$. Such findings do not, however, directly require quantum brain dynamics. These are based mainly on the idea that cognitive processing can be viewed as a superposition of many outcomes, and on the observation that the temporal order of mental states viewed as "self-measurements" can affect decision-making. Indeed, we can agree that when an experimenter makes measurements to obtain some observables about the external world, his/her brain operates exclusively within its own dynamics and generate conscious states as results of self-measurement. Authors suggest many psychological findings that agents can learn and make decisions that are not governed exclusively by classical logic. In modern neuroscience, it is commonly accepted that all conscious abilities emerge from relevant brain processes. Hence, quantum-like cognition models - if those are not of abstract interest-have to account for quantum logic utilized by neural networks in unambiguous causal ways. If noncommutativity of cognitive decisions is simply related to the arrow of time, this should be rather ubiquitous in human thinking. Nonetheless, some physical processes in neural networks must be still required to explain the irreversibility of time.

Instead, Orch OR is founded on quantum computations in microtubules during integration phases in dendrites and cell bodies of neurons linked by gap junctions. Leaving aside the Gödel theorem, Penrose and Hameroff ask: How do unicellular organisms (like Protozoans) that are full of microtubule nanotubes in their cytoskeleton but, clearly, with no neural network manifest purposeful (intelligent) behavior? Their crucial thesis is that that consciousness depends on quantum computations where large-scale entanglements among many qubits would enable complex and highly efficient parallel processing in microtubules not in synapses. ${ }^{1}$

In this way, quantum entanglement distributed over many brain regions by influencing the release of neurotransmitters and the firing of synapses between neurons might be viewed like a

\footnotetext{
${ }^{1}$ Quantum parallel computing (if possible as microtubule vibrations in megahertz) might be favored by evolution if only its adaptive benefits for cognition and behavior under selection were higher than its energy cost in neural performance.
} 
spooky action at a distance. A quantum computation terminating by $\mathrm{OR}$ as the gravitation-induced collapse could select a particular set of tubulin states to trigger axonal firings, and, thus, correspond to elementary acts of consciousness. Penrose notes that quantum Darwinism does not explain the collapse (OR) of a quantum system if isolated from the environment. For example, the whole universe might be such a quantum system beyond any environment and described by the wavefunction as it is taken for Many Worlds. Instead, the gravitational collapse should result immediately from spacetime.

The active role of consciousness, i.e., free will properly, should then emerge by the nonlocal quantum backward-time referral, resulting from entanglement aspects of Orch OR to rescue consciousness from its unfortunate characterization as a passive epiphenomenal spectator [15]. One can lightly find a mysterious separation of consciousness from the brain in this scenario. Let alone gravitational yet-not-uncovered effects in OR, there is something of dualism in this explanation of free will as if emerging ahead of brain activity. On the other hand, in regard to 'protoconscious' events intrinsic to the universe as precursors of consciousness, the Orch OR shares some elements with panpsychism.

Suppose, the Orch OR is true in prescribing free will to consciousness. How might consciousness be separated even for a moment from the brain to make use of nonlocal binding and quantum backward-time referral, thereby having causal power over neural dynamics? Unless Descartes' mind-matter dualism applies, consciousness as being derived from the brain cannot do it by its physical origin. Nor can consciousness gain an advantage over the brain from a physics-driven modification of dualism - Bohr's idea of complementarity of physics and psyche advocated by Pauli [44] and encapsulated ultimately in Wheeler's Participatory Anthropic Principle (PAP) "Observers (i.e. consciousness - S.Y.) are necessary to bring the universe into being" [35]. On the other hand, if related to Whitehead's becoming, the complementarity and PAP both may also be related to panpsychism.

\subsection{Beck-Eccles Model}

I consider Beck and Eccles [19] approach as the most realistic hypothesis of how QM might play a role in brain processes to account for volitional mechanisms. It refers to particular mechanisms of information transfer at the synaptic cleft. The central idea here is that synaptic transmission represents a typical (quantum) probability state in which the total number of vesicles available for exocytosis is critical for an all-or-none response of neuronal firing [45]. The trigger mechanism proposed by them is based on electron transfer between biomolecules at single synapses undergone then quantum stochastic resonance to achieve ordered patterns at the macro-level of neural networks from fundamentally random synaptic processes. The coherence of neural processes (the binding problem) for generating conscious unitary experience is imposed upon critical nonlinear dynamics capable of self-organization at classical timescales.

Although Eccles maintained "interactionist dualism" very similar to views of Wheeler and Pauli, his metaphysical position did not follow immediately from the neurophysiological mechanism suggested by him. For neuroscience the question has rather to be this. Can the brain generate conscious states that are partly independent of the antecedent neural processes and, in general, the past of the whole universe? If the answer is "yes" then the brain can have a free volitional mechanism (quantum in origin) for processing corresponding neurocognitive states that are not 
completely predetermined from the past. Because of their indeterminism brain processes could not be computable in principle by a machine. This entails two consequences: (i) one's consciousness can never be cloned, and (ii) artificial consciousness has to be impossible to run on Al devices. There is no philosophical mind-body dualism there. Therefore, I relate the Beck-Eccles model to Quantum Darwinism rather than to Neumann-Wheeler dualism making consciousness a separate entity responsible for the wavefunction collapse.

\subsection{Stapp Model}

Stapp's main motivations for the quantum brain hypothesis are of the same kind as of Orch-OR to account for free will. To do so, he adopts Neumann-Wheeler dualism and Whitehead [32] metaphysics to provide an alternative to completely deterministic human volition. Stapp [33] argues that while the universe evolves over Whitehead's "becoming" and/or Heisenberg's duplex world, the mental effort of consciousness can protract the lifetime of neuronal ensembles that represent the templates for action due to quantum Zeno-type effects. Within the quantum framework, a person's acquired knowledge of reality is not a faithful representation of the preexisting properties of the observed system but is, instead, an output of a dynamical probing process initiated by the observer. The universe responds to the observer in classical Yes-No terms. The origin of conscious free will is placed in a synaptic cleft like the Beck-Eccles approach, but where calcium ions should trigger the release of neurotransmitters and somehow split the whole brain into different classically describable components that specify alternative possible courses of action. No plausible neurophysiology is suggested. Again, how might consciousness choose actions to avoid the "separation sin?"

\subsection{Freeman-Vitiello Model}

The dissipative model of Vitiello and Freeman [46] describes the brain as a fully integrated macroscopic quantum system that transforms sensory signals into consciousness almost instantly. This is based on the well-known observation that brain dynamics exhibits distinctive synchronization patterns in the range of $12-80 \mathrm{~Hz}$ in time duration about $80-120 \mathrm{~ms}$ with an abrupt shift. Authors conclude that an external stimulus acts on the brain as a trigger for the spontaneous breakdown of the symmetry with long-range correlations established by the coherent condensation of the Nambu-Goldstone bosons responsible for the formation of amplitude-modulated synchronized oscillations in the neocortex. The density of the condensed bosons provides a measure of the degree of coherence. In contrast to Neumann-Wheeler dualism, the model rejects a possibility of separation between mental activity and brain activity: The brain modes and the mental (Double) modes appear entangled in the coherent states through which the activity evolves. There are no two entities, no dual level of existence, matter and mind, but only one undividable entity [47]. Nevertheless, no neurophysiological mechanism is proposed to provide free volition. Instead, the authors argue for certain hidden order similar to Bohmian wholeness that underlies chaotic, complex, and seemingly random behavior of brain dynamics. Thus, I relate this model to a superdeterministic account. 


\subsection{Georgiev Model}

Another view on brain dynamics is of Georgiev [48] who associates private consciousness (mind) directly with quantum information that cannot be observed by the no-cloning theorem. This information pervades the observable brain by means of quantum tunneling in voltage-gated ion channels via SNARE proteins that drive the exocytosis of synaptic vesicles [49]. Consciousness is postulated to be continuous (except the states where it vanishes in sleep, anesthesia, and coma) and operating at the quantum level on the picosecond timescale despite a wealth of neuroscientific evidence on priming, masking, and attentional blink that conscious experience is discrete with an unconscious preparation period of about 300-400 ms e.g. [50-52].

Unlike Beck-Eccles exocytosis and Orch OR where quantum indeterminism is only employed either for a random outcome to trigger off the neural nonlinear dynamics at classical timescales or as a non-unitary, non-computable quantum state reduction in spacetime, both causing conscious discrete states, the quantum mind model identifies the mind with the unitary evolution of the wavefunction $\Psi$ so that conscious continuous experiences originate as physical solutions of the Schrödinger equation. Instead of unconscious processes, this assumes the existence of multiple experiences in the subcortical areas to which, however, the conscious "I" has no concurrent access.

After all, I put Georgiev's model upon Heisenberg's duplex world.

\subsection{Classical Models}

Classical theories of consciousness are obviously grounded on brain dynamics that are initially assumed to be completely deterministic.

The integrated information theory (IIT) of Tononi [53] defines consciousness as integrated information specified by the irreducible causal mechanisms in the brain over cause-effect repertoires. According to IIT, one's free choice is guaranteed by this maximal irreducibility of mechanisms in one's brain [54]. To the best, free will is referred here to one's individuality and the uniqueness of the environment changing every time so that no state of both the brain and the environment can occur twice. This is drawn on a psychological rather than a physical aspect of free volition. In particular, the theory allows for the possibility of multiple local complexes, each with its maximum $\Phi$ and coexisting within a single system (not necessarily the brain). In principle, the theory agrees that even a mitochondrion inside a biological cell can maintain some level of consciousness [55]. Thus, the unconscious, from the perspective of the major complex in the brain, may be attributed to the presence of minor complexes there.

In contrast, the global workspace theory (GWT) is scale-dependent and emphasizes the dynamical core concentrated in the thalamocortical system to broadcast information over all brain regions $[56,57]$. Consciousness fades (in sleep, anesthesia, and coma) if synchronization of neural activities in the dynamical core is reduced or interrupted. Volition is not discussed directly but implicitly embedded in the broadcasting. Another dominant theory, predictive processing theory (PPT) is more involved with studying cognition [58] and treats the brain to be a Bayesian machine that follows the free energy principle (FEP). Consciousness is derived from cognition function, whereas volition is simply referred to active processing without explaining its physical foundations.

Thus, free will of these theories is "compatibilist" as it is assumed per se in physics, psychology, and social sciences. Humans are said to be consciously free in everyday activities, rational immediate agency, pursuing their long-term objectives, and, after all, in making science: they can 
decide freely how to prepare a scientific experiment as, for example, that of Bell-type or Libet-type experiments, or what to write in their papers.

Although IIT and PPT/FEP assume panpsychism [59] or its neutral variations [60], which are similar to Leibniz's notion of proto-conscious particle-like monades, I relate them to a superdeterministic account as being indifferent to quantum effects in brain dynamics.

\section{Superdeterminism vs. Randomness}

When we refer to "matter" we do it as if we knew exactly what it is. But the word "matter" is not better and explains no more than the word "psyche" to have an advantage over the latter, besides our own linguistic preference. This is a reason why the term "physicalism" had been accepted by scientists instead of "materialism" in philosophy. However, the fundamental property of physicalism is determinism or, ultimately, superdeterminism. At this point, materialism seems to bluntly discard a veil of mystery from consciousness thereby ruling out dualism, not to mention idealism. Brains are only sophisticated deterministic machines.

Superdeterminism is a hypothesis that everything in Nature is predetermined from the past, free will is impossible, and the universe is a computation running automatically by natural laws from the initial conditions of the universe. It amounts to old-fashioned fatalism: we do what the universe wants us to do. If we combine Big Bang cosmology, the second law of thermodynamics with its inevitable growth of entropy (disorder) in time, and the anthropic arguments [61], we can come to the question: why and how should conscious observers appear in such a universe? The answer is that the universe had started with the Big Bang singularity at an incredibly ordered state whose entropy should be just zero so that all the future events in the universe, including the origin of life, the evolution of species, and, in particular, our birth, life, and death, had been uniquely encoded in the initial conditions of the Big Bang. Superdeterminism admits that we are not merely deterministic machines, but each of us is a machine designed from the beginning of time [25].

Why should then those conscious machines be favored by evolution if having no advantage over simpler unconscious systems, yet deprived of free volition? It happens that superdeterminism leads to cosmic conspiracies [59] in the quantum mechanical EPR experiments involving conscious observers to make their experimental settings at will. Namely, to avoid random collapse after measurements causing nonlocal correlations between entangled particles at a distance, the universe should know ahead how those conscious observers would prepare experiment to make particular measurements. One should then seriously agree that humans and all animals are machines designed in the Big Bang. Logically, thus, if brains are no more than conscious computers deprived of free volition to decide their way, every particular consciousness turns out to be a "soul" designed from the beginning of time. On the other hand, the very term "consciousness" loses any meaning and becomes empty enough to be discarded as an illusion occupying some physical bodies called brains. Although suggested to justify materialism, superdeterminism is not a valid alternative of idealism at all; instead, it leads ultimately to Newtonian deism or creationism (although, most likely, neither Newton nor creationists would deprive human creatures of free will).

In other words, superdeterminism decides the mystery of consciousness by pushing all the mystery back to the origin of the universe. Upon closer examination, materialism appears to be even more mystical than dualism. Nevertheless, superdeterminism helps to understand that the problem of free volition comes to be crucial to the nature of consciousness. In fact, religion had met 
the problem many centuries ago. Humans might not be responsible for their actions if they had not free volition to do better than it was determined by God's providence. The only way for religious thought to reconcile providence (i.e., causal order) with moral responsibility of immortal souls was to agree that God had admitted free volition into the universe. Leaving aside dualism, free volition might not be possible without randomness in the universe. As a result, superdeterminism challenges not only free will but the origins of life in the universe (Figure 1).

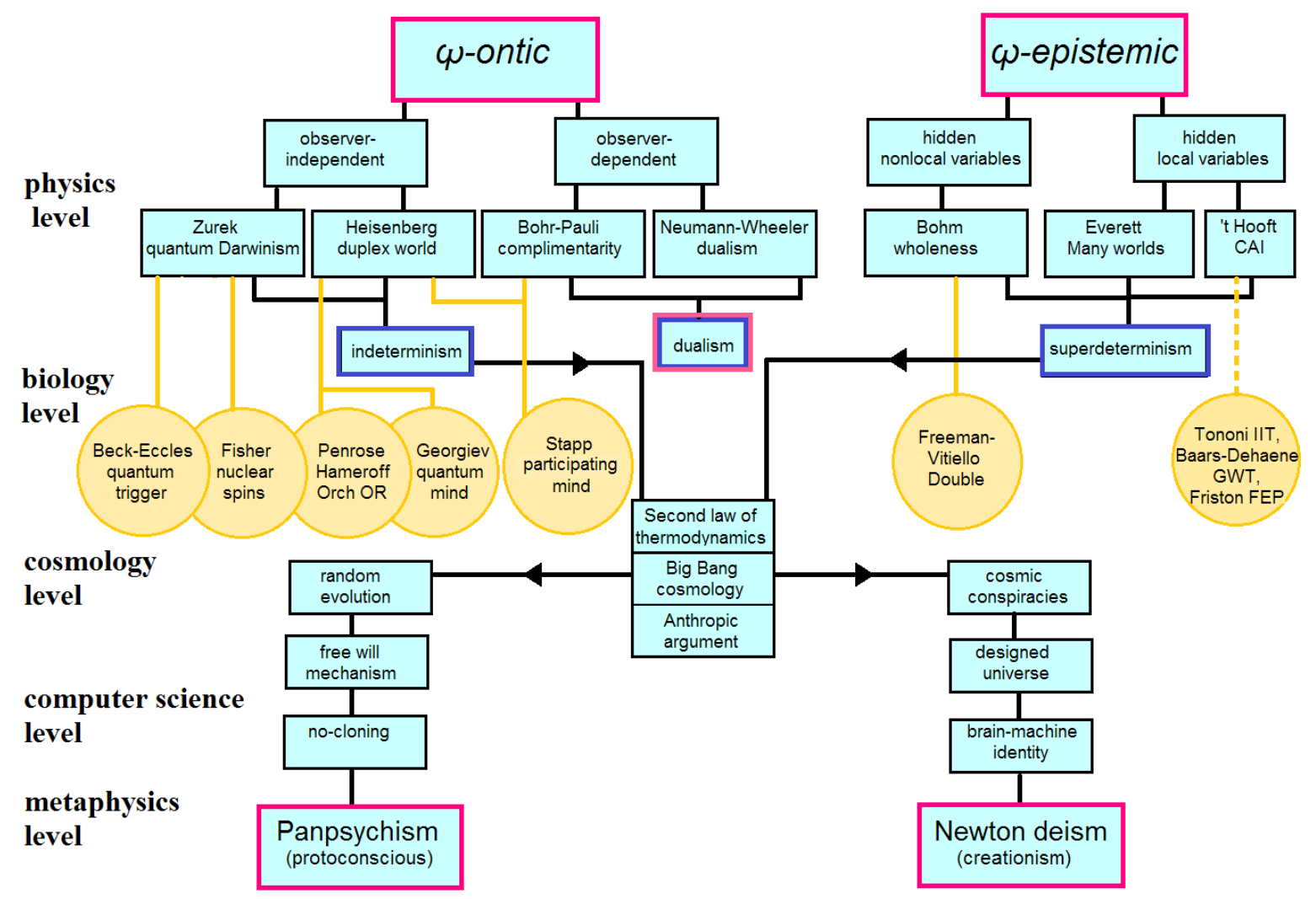

Figure 1 Quantum brain hypothesis. The most known quantum brain models are derived from corresponding interpretations of QM. Those can be then classified over different scientific domains from physics to cosmology and computational neuroscience ( $\mathrm{Al}$ and Turing test) to metaphysics. Classical theories of consciousness such as IIT, GWT, FEP (Bayesian brain), and others are indifferent to quantum effects in brain dynamics, and can, in principle, be related to a superdeterministic account.

So, modern Darwinism holds that the evolution of life is driven by random (quantum in origin) genetic mutations that underwent natural selection. Thus, if we disagree with all of aforesaid for one or another reason, we need to reconcile quantum randomness with brain dynamics to account for free will. First, Nature should somehow use quantum effects of genetic mutations driving evolution at cellular and molecular levels in order to give rise to volitional abilities (over reflex mechanisms) at neural networks. In principle, it would be well enough to say with confidence that some quantized micro-events in the brain should be not averaged as noise in classical spatiotemporal scales but amplified across multiple levels in neural networks as macro-events that had not been predetermined completely from the past (including previous states of both the brain 
and its environment) but might influence the present state of the brain. This is a necessary and likely sufficient condition for the brain (not consciousness) to have free volition.

In doing so, we need to postulate some neurophysiological free volition mechanism, quantum in origin, which should have evolved from primary (rapid and random) reflexes of the simplest organisms and then inherited by the oldest domain of neural networks, the brainstem. This is involved in many homeostatic unconscious processes: a baroreflex of blood pressure, breathing, yet, famously engaged in spontaneous arousal, i.e., returning consciousness after sleep and anesthetized states. The random contributions of the mechanism should be cognitively constrained in the thalamocortical system via predictive processing to cause the ultimate response of the brain that was rationally motivated. Such a response would not be completely predetermined from the antecedent brain dynamics.

However, we can never know with confidence that anything is truly random. The most we can ever say is that a deterministic pattern we seek for is beyond our experimental capabilities. This is just the reason why Bell-certification is suggested in cryptographic applications of quantum effects [62]. Yet, many macro-events in the nonlinear stochastic dynamics of the brain and consequent mental phenomena (e.g. as those in quantum-like cognition [40-43]) that are involved with quantum-inspired effects can be explained classically as well. Since the distinction between genuine (ontic) randomness and stochastic probabilistic (epistemic) patterns is elusive and algorithmically sophisticated, the mechanism should be Bell-certified.

The Beck-Eccles [19] quantum trigger in a synaptic cleft (possibly, via SNARE proteins [49]) or Fisher's [20] processing with nuclear spins in Posner molecules influencing neuronal (classical) firing, look most promising and reliable candidates for the mechanism. This could lightly account for neural stochastic dynamics free of predetermination. After all, such a mechanism might provide "freebits" [17] and guarantee no-cloning of consciousness, thereby forbidding the scenarios where a particular consciousness might be cloned to generate multiple personalities running simultaneously on digital computers.

\section{Discussion}

Strikingly, many neuroscientists, physicists, and computer scientists speaking so much about consciousness have almost nothing said about free will viewed likely by them as too ambiguous and metaphysical to discuss meaningfully. Meanwhile, the free will problem has to be fundamental to the nature of consciousness and cognition, especially in regard to the computational theory of mind and the brain-machine metaphor. Quantum effects in the brain must naturally be involved in the problem.

It is often said that if even quantum processes might revive over rapid decoherence, the quantized event should be statistically averaged by the second law of thermodynamics [63]. First, the law does not indulge the evolution of life in general. Why should complex biosystems arise once from the less-ordered medium and become more and more ordered over time under entropy growth? Of course, evolution does not violate the law, but that in turn does not explain life. Second, the brain is just evolved as the most complex system in the universe to sidestep commonality. So, Tegmark [64] contends that the only distinction one might (arguably) find between a classical computer and consciousness is the information integration in the brain as it is, for example, presented in IIT $[53,64]$ that also ignores the free will problem. 
On the other hand, Tegmark concedes that Quantum Darwinism [37, 38] that emphasizes the role of environment-induced decoherence in copying information about the redundant quantum states may be relevant to the evolution of consciousness from the simplest forms in a way similar to the fitness principle fundamental to all biological species. If an organism can attain an advantage in adaptation over quantum sensitivity, then with time natural selection has a chance to engineer the necessary biochemical mechanisms to exploit these effects [65].

It is commonly accepted that consciousness emerges causally from neural correlates of the brain as the Jamesian irreversible stream of states in time [39]. Thus, the idea of free will that might somehow admit consciousness to be self-made by arriving at a state just chosen by it ahead of time is incompatible with aforesaid. The only natural mechanism that might account for indeterminism of free will lies in a quantum domain. To solve this problem, one should assume that the brain can classically process those states generated by the neurophysiological free choice mechanism. Such a mechanism would break up rigid causal chains of the universe and provide living systems with flexible stimulus-reaction repertoires of possible actions. If so, the stream of consciousness should be initially free-willed by the brain itself whereas consciousness might never have power over it. There is a reason to think that the fundamental mechanisms of volition should appear in the brain evolution even earlier than consciousness and cognition, requiring much more neural resources.

It is often argued that epiphenomenal consciousness possessing no causal potency would be useless. Nature should then have no reason to evolve consciousness gradually from bacteria to humans. The problem can be solved by assuming that conscious discrete experience is derived from cognitive continuous processes, e.g., as ultimate decisions of predictive (Bayesian) processing, thereby making consciousness like a mental interface between the internal cognitive world of the brain and the external world around (Graphical abstract). Thus, not consciousness but cognition from which it emerges is the main purpose of evolution insofar as just mental properties have to be favored by natural selection in the struggle for life. The more the brain is cognitively adapted to the environment, the higher its conscious properties.

While the brain mechanisms that enable the capacity of free-willed actions remain unclear, it seems plausible to agree that the brain evolution might be of little use in survival without the ability to make a free choice under selection pressure. From the evolutionary point of view, the adaptive advantages of free volition might be seen as an extremely valuable acquisition for the flourishing of life because the ability to maneuver would crucially help even relatively simple organisms in surviving and reproducing [66].

Because of the high sensitivity of nonlinear complex systems to initial conditions, quantum fluctuations may be essential in living systems. Increasing evidence suggests that biological evolution is able to exploit quantum phenomena [1-3] in wet protein environments conducive to the survival of quantized events [67]. So, in systems of photosynthesis, coherent vibrational motions that do not relax quickly and whose fluctuations cannot be described classically may be seen as an internal quantum mechanism controlling energy transfer and storage in cells $[4,5]$ and, hence, in neural structures $[49,68]$.

It also means that though neuronal computations and information storage are classical in the brain, its more detailed activity might be sensitive to quantum fluctuations. Instead of being averaged out on classical spatiotemporal scales [69], these might be chaotically amplified and orchestrated to be relevant to behavioral manifestations. In this way, quantum brain dynamics 
might influence neuronal computations leading the brain (not consciousness) to a self-initiated action that had not been completely controlled from a distant past.

\section{Conclusions}

The superdeterministic view provided by materialism assumes that not only free will but consciousness itself does not exist at all. Upon a close examination, however, this option requires a much more radical assumption than panpsychism does, namely, Designer outside the universe to account for the origins of the universe. Thus, if logic and scientific evidence enforce us to choose between dualism and panpsychism, the idea that the universe consists of "proto-conscious" processes that might naturally evolve living high-ordered systems from low-ordered environments (not despite the second law of thermodynamics), and after all give rise to conscious brains endowed with free volition, the Occam's razor tells us that dualism only multiplies entities without explaining anything, thereby making panpsychism much more preferable. Pierre Teilhard de Chardin had yet noted (anticipating the anthropic arguments) that the idea of mind-like evolution leads to panpsychism: The creative activity of matter can be explained only if mentality is present in the world at its very beginning. In other words, either the universe is a computation going on its own by actions of law from the initial conditions created and established by God from outside the Big Bang, or the universe itself is something like God which every consciousness takes part in.

I would like to finish on a personal note. If the only alternative to panpsychism is deism of Newton, Laplace, and Einstein with consequent fatalism where Creator had decided everything, I tend to choose the former. I am ready to agree with Spinoza, Leibniz, Whitehead, and others that the universe is proto-conscious (Prigogine [70]) and even mindful (Stapp [71]), and we humans and any conscious beings are its advanced parts; but I disagree that someone had designed the universe as a nonlocal Wholeness (Bohm [26]) or as a local Cellular Automaton ('t Hooft [27]) predetermined completely by the initial conditions of the Big Bang, and each of us is literally a machine deprived of any kind of volition to make our ways better than those placed by the Designer.

\section{Author Contributions}

Sergey B. Yurchenko did all the research work of this study.

\section{Competing Interests}

The author declares that no competing interests exist.

\section{References}

1. Engel GS, Calhoun TR, Read EL, Ahn TK, Mančal T, Cheng YC, et al. Evidence for wavelike energy transfer through quantum coherence in photosynthetic systems. Nature. 2007; 446: 782-786.

2. Collini E, Wong CY, Wilk KE, Curmi PM, Brumer P, Scholes GD. Coherently wired light-harvesting in photosynthetic marine algae at ambient temperature. Nature. 2010; 463: 644-647.

3. Gauger EM, Rieper E, Morton JJ, Benjamin SC, Vedral V. Sustained quantum coherence and entanglement in the avian compass. Phys Rev Lett. 2011; 106: 040503.

4. O'Reilly EJ, Olaya-Castro A. Non-classicality of the molecular vibrations assisting exciton energy transfer at room temperature. Nat Commun. 2014; 5: 1-10. 
5. Chenu A, Scholes GD. Coherence in energy transfer and photosynthesis. Annu Rev Phys Chem. 2015; 66: 69-96.

6. Fried I, Mukamel R, Kreiman G. Internally generated preactivation of single neurons in human medial frontal cortex predicts volition. Neuron. 2011; 69: 548-562.

7. London M, Roth A, Beeren L, Häusser M, Latham PE. Sensitivity to perturbations in vivo implies high noise and suggests rate coding in cortex. Nature. 2010; 466: 123-127.

8. Houweling AR, Doron G, Voigt BC, Herfst $\amalg$, Brecht M. Nanostimulation: Manipulation of single neuron activity by juxtacellular current injection. J Neurophysiol. 2010; 103: 1696-1704.

9. Fujisawa $S$, Matsuki N, Ikegaya $Y$. Single neurons can induce phase transitions of cortical recurrent networks with multiple internal states. Cereb Cortex. 2006; 16: 639-654.

10. Cheng-yu TL, Poo MM, Dan Y. Burst spiking of a single cortical neuron modifies global brain state. Science. 2009; 324: 643-646.

11. Tanke N, Borst JG, Houweling AR. Single-cell stimulation in barrel cortex influences psychophysical detection performance. J Neurosci. 2018; 38: 2057-2068.

12. Jedlicka P. Revisiting the quantum brain hypothesis: Toward quantum (neuro)biology? Front Mol Neurosci. 2017; 10: 366.

13. Rosu HC. Essay on mesoscopic and quantum brain. Metaphys Rev. 1997; 3: 1-12.

14. Georgiev DD. Quantum information and consciousness: A gentle introduction. Boca Raton: CRC Press; 2017.

15. Hameroff S. How quantum brain biology can rescue conscious free will. Front Integr Neurosci. 2012; 6: 93.

16. Tognoli E, Kelso JS. The metastable brain. Neuron. 2014; 81: 35-48.

17. Aaronson S. The ghost in the quantum Turing machine. In: The once and future Turing: Computing the world. Cambridge: Cambridge University Press; 2016. p.193-294.

18. Hameroff $S$, Penrose R. Consciousness in the universe: A review of the 'Orch OR' theory. Phys Life Rev. 2014; 11: 39-78.

19. Beck F, Eccles J. Quantum processes in the brain: A scientific basis of consciousness. Cogn Stud. 1998; 5: 95-109.

20. Fisher MP. Quantum cognition: The possibility of processing with nuclear spins in the brain. Ann Phys. 2015; 362: 593-602.

21. Conway J, Kochen S. The strong free will theorem. Not Am Math Soc. 2009; 56: 226-232.

22. Libet $B$. Unconscious cerebral initiative and the role of conscious will in voluntary action. Behav Brain Sci. 1985; 8: 529-539.

23. Haynes JD, Sakai K, Rees G, Gilbert S, Frith C, Passingham RE. Reading hidden intentions in the human brain. Curr Biol. 2007; 17: 323-328.

24. Barlas Z, Obhi SS. Freedom, choice, and the sense of agency. Front Hum Neurosci. 2013; 7: 514.

25. Yurchenko SB. The importance of randomness in the universe: Superdeterminism and free will. Axiomathes. 2020; 1-26. doi:10.1007/s10516-020-09490-y

26. Bohm D. Wholeness and the implicate order. London: Routledge \& Kegan Paul; 1980.

27. ' $t$ Hooft G. The cellular automaton interpretation of quantum mechanics. New York: Springer; 2016.

28. Everett H. Relative state formulation of quantum mechanics. Rev Mod Phys. 1957; 29: 454-462.

29. Bell JS. Speakable and unspeakable in quantum mechanics. Cambridge: Cambridge University Press; 1993. 
30. Wootters WK, Zurek WH. The no-cloning theorem. Phys Today. 2009; 62: 76-77.

31. Heisenberg W. Physics and philosophy. New York: Harper; 1958.

32. Whitehead AN. Process and reality. New York: Process \& reality; 1929.

33. Stapp HP. Whitehead, James, and the ontology of quantum theory. Mind Matter. 2007; 5: 83109.

34. von Neumann J. Mathematical foundations of quantum mechanics. Princeton: Princeton University Press; 1955.

35. Wheeler JA. Information, physics, quantum: The search for links. In: Complexity, entropy, and the physics of information. Boca Raton: CRC Press; 1990. p.3-28.

36. Bohm DJ. A new theory of the relationship of mind and matter. Philos Psychol. 1990; 3: 271286.

37. Zurek WH. Quantum darwinism. Nat Phys. 2009; 5: 181-188.

38. Riedel CJ, Zurek WH, Zwolak M. The objective past of a quantum universe: Redundant records of consistent histories. Phys Rev A. 2016; 93: 032126.

39. Yurchenko SB. Can 'theory of everything' be global theory of consciousness? Ontology and psychodynamics of I-observer. NeuroQuantology. 2017; 15: 118-131.

40. Busemeyer JR, Wang Z, Townsend JT. Quantum dynamics of human decision making. J Math Psych. 2006; 50: 220-241.

41. Kornmeier J, Bach M. Ambiguous figures - What happens in the brain when perception changes but not the stimulus? Front Hum Neurosci. 2012; 6: 1-23.

42. Atmanspacher H, Filk T. The Necker-Zeno model for bistable perception. Top Cogn Sci. 2013; 5 : 800-817.

43. Khrennikov A. Quantum-like model of cognitive decision making and information processing. Biosystems. 2009; 95: 179-187.

44. Pauli W, Enz CP, von Meyenn K. Writings on physics and philosophy. Berlin: Springer; 1994.

45. Fisher MP. Are we quantum computers, or merely clever robots? Int J Mod Phys B. 2017; 31: 1743001.

46. Freeman WJ, Vitiello G. The dissipative quantum model of brain and laboratory observations. Electr J Theor Phys. 2007; 4: 1-18.

47. Freeman WJ, Vitiello G. Matter and mind are entangled in two streams of images guiding behavior and informing the subject through awareness. Mind Matter. 2016; 14: 7-24.

48. Georgiev DD. Quantum information theoretic approach to the mind-brain problem. Prog Biophys Mol Biol. 2020; 158: 16-32.

49. Georgiev DD, Glazebrook JF. The quantum physics of synaptic communication via the SNARE protein complex. Prog Biophys Mol Biol. 2018; 135: 16-29.

50. Sergent $C$, Baillet $S$, Dehaene $S$. Timing of the brain events underlying access to consciousness during the attentional blink. Nat Neurosci. 2005; 8: 1391-1400.

51. Seitz AR, Kim D, Watanabe T. Rewards evoke learning of unconsciously processed visual stimuli in adult humans. Neuron. 2009; 61: 700-707.

52. Drissi-Daoudi L, Doerig A, Herzog MH. Feature integration within discrete time windows. Nat Commun. 2019; 10: 4901.

53. Tononi G. Consciousness as integrated information: A provisional manifesto. Biol Bull. 2008; 215: 216-242. 
54. Tononi G. On the irreducibility of consciousness and its relevance to free will. In: Is science compatible with free will? New York: Springer; 2013. p.147-176.

55. Tononi G, Koch C. Consciousness: Here, there and everywhere? Phil Trans R Soc B. 2015; 370 : 20140167.

56. Baars BJ. The conscious access hypothesis: Origins and recent evidence. Trends Cog Sci. 2002; 6: 47-52.

57. Dehaene S, Naccache L. Towards a cognitive permanence of consciousness: Basic evidence and a workspace framework. Cognition. 2001; 79: 1-37.

58. Friston KJ, Daunizeau J, Kilner J, Kiebel SJ. Action and behavior: A free-energy formulation. Biol Cybern. 2010; 102: 227-260.

59. Gallicchio J, Friedman AS, Kaiser DI. Testing Bell's inequality with cosmic photons: Closing the setting-independence loophole. Phys Rev Lett. 2014; 112: 110405.

60. Friston KJ, Wiese W, Hobson JA. Sentience and the origins of consciousness: From cartesian duality to Markovian monism. Entropy. 2020; 22: 516.

61. Barrow JD, Tipler FJ. The anthropic cosmological principle. Oxford: Clarendon Press; 1986.

62. Pironio S, Acín A, Massar S, de La Giroday AB, Matsukevich DN, Maunz P, et al. Random numbers certified by Bell's theorem. Nature. 2010; 464: 1021-1024.

63. Tegmark M. The importance of quantum decoherence in brain processes. Phys Rev E. 2000; 61: 4194-4206.

64. Tegmark M. Consciousness as a state of matter. Chaos Solitons Fractals. 2015; 76: 238-270.

65. Lloyd S. Quantum coherence in biological systems. J Phys Conf Ser. 2011; 302: 012037.

66. Brembs B. Towards a scientific concept of free will as a biological trait: Spontaneous actions and decision-making in invertebrates. Proc Royal Soc B. 2011; 278: 930-939.

67. Brookes JC. Quantum effects in biology: Golden rule in enzymes, olfaction, photosynthesis and magnetodetection. Proc Royal Soc A. 2017; 473: 20160822.

68. Kariev AM, Green ME. Quantum calculations on ion channels: Why are they more useful than classical calculations, and for which processes are they essential? Symmetry. 2021; 13: 655.

69. Koch C, Hepp K. Quantum mechanics in the brain. Nature. 2006; 440: 611.

70. Prigogine I, Stengers I. The end of certainty: Time, chaos, and the new laws of the nature. New York: The Free Press; 1997.

71. Stapp HP. Mindful universe: Quantum mechanics and the participating observer. Berlin: Springer; 2007. 


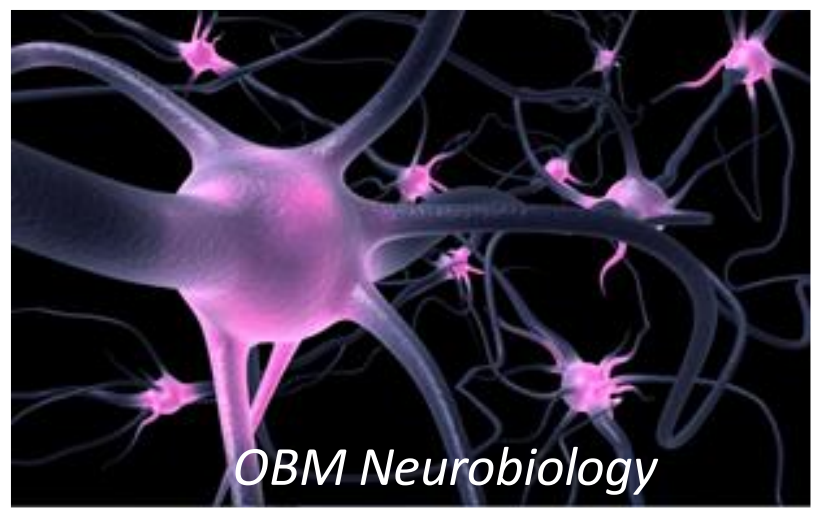

Enjoy OBM Neurobiology by:

1. Submitting a manuscript

2. Joining volunteer reviewer bank

3. Joining Editorial Board

4. Guest editing a special issue

For more details, please visit:

http://www.lidsen.com/journals/neurobiology 